\author{
Joanna P. Anosova \\ Astronomical Observatory \\ Leningrad State University \\ Bibliotechnaya pl.l \\ 198904 Leningrad Petrodvorets USSR
}

\begin{abstract}
A statistical criterion has been proposed to reveal the random and physical clusterings among stars, galaxies and other objects. This criterion has been applied to the galaxy triples of the list by Karachentseva, Karaschentsev and Scherbanovsky, and the double galaxies of the list by Dahari where the primary components are the seyfert galaxies. The confident physical, probable physical, probable optical and confident optical groups have been identified. The limit difference of radial velocities of components for the confident physical multiple galaxies has also been estimated.
\end{abstract}

\title{
CLASSIFICATION OF GROUPS
}

The study of the structure of some class of gravitating bodies - the field of the stars or galaxies, as well as the simulated field of the $\mathrm{N}$-body systems at some time of their evolution - is a search for the subsystems of different nature the groups and the clusters with different numbers of members and the various forms, the chains, the volds, etc. It is possible to 
propose (Anosova 1986, 1988) the following classification of groups of gravitating objects (stars, galaxies or their groups, clusters, etc.) in order to detect these groups objectively:

(I) The groups in which all or a few compoents are by chance in the group region of the phase space; for these groups the criterion of isolation from the background object in the phase space is not fulfilled; one may qualify such groups as chance groups of objects or optical systems;

(II) The groups which are not such chance groups; their components have some common features; however, the gravitational forces between components are small or approximately equal to the regular forces of the environment; for these groups the criterion of isolation from the background objects in the coordinate space is not fulfilled; one may qualify such groups as non-chance groups of objects. One might divide these groups into two clsses:

(IIa) groups which are completely isolated in velocity space (e.g., moving star clusters);

(IIb) groups which are partially isolated in velocity space (e.g., in one or two of the velocity components, like some of the well-known Eggen's kinematical groups);

(III) the groups for which the criteria of isolation from the background objects are fulfulled in the coordinate space; in these groups the irregular gravitational forces between components (which are $\mathrm{n}$ in total number) essentially exceed the regular forces of the environment; one may qualify such groups as multiples with a physical connection between components. 
(III-1) the multiple systems in which there is not a dynamical connection among all the components or a part of them; their total energy is non-negative $E>0$. One might also divide such systems into two subclasses:

(III-1a) multiple systems in which there is no genetic connection among all the components or a part of them; all those $\mathrm{n}$ objects in a system, which are single, rapidly pass by each other or a number $s(s<n)$ of single components and subsystems inside this system rapidly pass by each other;

(III-1b) multiple systems in which there is a genetic community of components but also there is a 'recession' of components $(E>0) ;$ e.g., according to the well-known concept of Ambartsumian;

(III-2) the multiples in which there is a dynamical connection between components, their total energy is negative $\mathrm{E}<$ $0 ;$ one might also divide such systems into two subclasses:

(III-2a) multiple systems in which there is no genetic community inside any part of components: $s(s<n)$ of single components and subsystems with a smaller multiplicity inside a system slowly $(E<0)$ pass by each other;

(III-2b) multiples in which there is a genetic community amongst all components (a co-formation), such systems may belong to one of the two dynamical types:

$$
\begin{aligned}
& \text { (III-I)* dynamically stable multiples; } \\
& \text { (III-II)* dynamically unstable ones. }
\end{aligned}
$$




\section{CRITERIA OF CLASSIFICATION}

In order to recognize the systems of type I - the optical systems, or chance groups of objects, among the observed multiple stars or galaxies one can use a statistical criterion (see Anosova 1987) which consists in obtaining a probability $P$ of this system being such one, and in estimating an expectation EX of the number of such chance groups in some phase volume $\Sigma$. One assumes for a calculation of $\mathrm{P}$ and Ex that the objects under study are randomly distributed inside the $\Sigma$. Generally speaking, this criterion may be generalized for every distribution of the phase density of objects. If the values $P$ and EX obtained are too big $(P \sim 1, E X \sim N / n$, where $N / n$ is the maximum number of groups with multiplicity $\mathrm{n}$ in the volume $\Sigma$ ), then this group is a probable chance group. Otherwise $(P \sim 0, E X \lesssim I)$, such a group is a probable non-chance group of objects and it belongs to the multiples of types II or III.

In order to recognize the groups of types II and III impartially one might use an analogous statistical criterion of isolation of the groups in the coordinate space. If such a criterion is not fulfilled, a group belongs to type II; otherwise, this multiple system is one with components connected physically.

The values $P_{n}$ and $E_{n}$ can be expressed as follows:

$$
P_{n}=C_{N-I}^{n-I} B^{n-I}(I-B)^{N-n}, E X_{n}=C_{N}^{n} B^{n-I}(I-B)^{N-n} ;
$$

$B=V(\sigma) / V(\Sigma)$, where $V(\sigma)$ and $V(\Sigma)$ are the volumes of $\sigma$ and $\Sigma, \sigma$ 
is the phase space, of the group of multiplicity $n, \Sigma$ is the phase space, of the whole sample of $\mathrm{N}$ objects, $\mathrm{N}=$ $(4 \pi / 3) R^{3} \nu, r$ - the radius of the spherical volume $\Sigma, \nu$-the mean number density of $\mathrm{N}$ objects in $\Sigma$. When applied to galaxies, $B$ has the following form

$$
B=0.25 \tan \left(\rho_{n} / 2\right)^{2}\left(r_{I} / R\right)^{3}\left[I-\left(r_{n} / r_{I}\right)^{3}\right] \text {, by } r_{n}<r_{I} \text {, }
$$

where $\rho_{n}$ is the angular separation between the galaxies $n$ and $I$; $r_{n}$ and $r_{I}$ are their line-of-sight distances (throughout this paper the Hubble constant $\mathrm{H}=75 \mathrm{~km} / \mathrm{s} / \mathrm{Mpc}$ is assumed). For obtaining the maximum estimate for $P_{n}$, we have taken $R=r_{I}$. If $r_{n}>r_{I}$, the indexes $I$ and $n$ change their positions by $r$ in the formula for $B$.

We shall use the above statistical criterion for analyzing membership in the list of triples by Karachentseva, Karachentsev and Scherbanovsky (1979) and in the double galaxies of the list by Dahari (1985). Table I presents the distributions of multiples $N(E X)$. Table I shows the numbers of the confident physical $(\mathrm{EX}<1)$ and probably physical (EX $<300)$ systems as well as the numbers of the probably chance $(300<\mathrm{EX}<1000)$ and confident chance (EX $>1000)$ groups of galaxies. An increase of the cutoff value $\mathrm{Ex}_{\mathrm{Cr}}=300$ may be caused by the internal dispersion of velocities of galaxies as well as by the uncertainties of the values.

One might also calculate by using the above statistical criterion the cutoff differences $\Delta \mathrm{v}_{\mathrm{cr}}$ of the radial velocities of galaxies inside the confident physical multiples of Ex $<1$, the 
Table 1. The distributions of the values EX for the triples (I) and the double galaxies (II).

\begin{tabular}{rrrrrrrrr}
\hline EX & $<1$ & $1-10$ & $10-100$ & $100-300$ & $300-500$ & $500-1000$ & $>1000$ \\
\hline I I & 10 & 5 & 16 & 13 & 3 & 3 & 33 \\
\hline
\end{tabular}

Table 2. The tolerant differences of the radial velocities of galaxies in the confident physical systems by $\mathrm{EX}<1$.

\begin{tabular}{|c|c|c|c|c|}
\hline $\mathrm{z}^{\mathrm{D}} \mathrm{kpc}$ & IO & 30 & 50 & 70 \\
\hline $\begin{array}{ll} & \mathrm{IO}^{-2} \\
2 & \mathrm{IO}^{-2} \\
3 & \mathrm{IO}^{-2} \\
4 & \mathrm{IO}^{-2}\end{array}$ & $\begin{array}{c}1000^{\star} \\
200 \\
50 \\
25\end{array}$ & $\begin{array}{r}150 \\
20 \\
5 \\
2\end{array}$ & $\begin{array}{r}60 \\
10 \\
2 \\
1\end{array}$ & $\begin{array}{l}30 \\
5 \\
1 \\
0.5\end{array}$ \\
\hline
\end{tabular}

different separations $D$ between the components and the Hubble distances $\mathrm{cz} / \mathrm{H}_{\mathrm{O}}$. Table 2 shows such results for the Dahari's double galaxies: by the smaller $D$ and $Z$ the value $\Delta v_{c r}=1000$ is possible, by an increasing $\mathrm{D}$ and $\mathrm{Z}$ the values $\Delta \mathrm{v}_{\mathrm{cr}}$ are strong decreasing.

CONCLUSION

After constructing the hierarchical structure of some field (e.g., Materne 1978), one might use the above statistical criterion in order to recognize objectively the subsystems of various nature with different numbers of members and the various forms. 
REFERENCES

Anosova, J.P. 1986, Astrofizika, 25, 2,297.

Anosova, J. P. 1987, Astrofizika, 27, 3,535.

Anosova, J. P. 1988, Astrophys. Space Sci., 142, 1-2,103.

Dahari, O. 1985, Astron. J., 90, 1772.

Karachentseva, V. E., Karachentsev, I. D., Sherbanovsky, A. L. 1979, Izv. Spec. Astrofiz. Obs., 11, 3.

Meterne, J. 1978, Astron. Astrophys., 63, 401 . 